Vietnam Journal of Mechanics, VAST, Vol.42, No. 1 (2020), pp. 87-94

DOI: https://doi.org/10.15625/0866-7136/14851

\title{
AMPLIFICATION OF USEFUL VIBRATION USING ON-OFF DAMPING
}

\author{
La Duc Viet ${ }^{1,2, *}$ \\ ${ }^{1}$ Institute of Mechanics, VAST, Hanoi, Vietnam \\ ${ }^{2}$ Graduate University of Science and Technology, VAST, Hanoi, Vietnam \\ E-mail: ldviet@imech.vast.vn
}

Received: 22 December 2019 / Published online: 17 March 2020

\begin{abstract}
This paper points out how much useful vibration can be extracted from a baseexcited oscillator, which is controlled by the on-off electrical damping. We studies the class of on-off electrical damping controller, which switches the damping level from high to low and back at fixed times every quarter of period. The problem reduces to the maximization of a single-variable function. This result can open the new direction to amplify the useful vibration using controllable dampings.

Keywords: vibration energy harvesting, frequency response, analytical optimization, on-off damping, optimal bound.
\end{abstract}

\section{INTRODUCTION}

A base-excited oscillator is a well-known model to extract useful vibration [1]. The optimization of electrical damping to extract maximum useful vibration has been extensively investigated in [1] and many references therein. If the damping is too large, the useful vibration is suppressed. Conversely, the too small electrical damping can make large vibration due to the resonance effect but this large vibration is useless. Those are the reason why the damping should be optimized to maximize the useful vibration.

To suppress the useless vibration, it is well-known that the performance of constant passive damping can be enhanced by the semi-active damping [2,3]. However, to amplify the useful vibration, semi-active damping is still not considered much. The switching technique applied to a piezoelectric harvester has been studied in some papers [4-6]. Some theoretical analyses of semi-active damping in energy harvesting have been presented in $[7,8]$.

In the field of vibration control, some papers pointed out how much useless vibration can be suppressed by a general controller of on-off damping [9-12]. In this paper, in the converse way, we derive clearly how much useful vibration can be extracted using on-off electrical damping. In literature, there is still no optimal solution (of constant damping) for the transient response. Therefore, for comparison, in this paper, only the

(C) 2020 Vietnam Academy of Science and Technology 
steady responses are considered. Energy extraction from transient response by on-off damping is indeed an interesting topic for the future studies. The found solution also gives a good direction to design the future practical on-off electrical damping controllers.

\section{PROBLEM STATEMENT}

Let us consider a base-excited SDOF oscillator with electromagnetic transduction as shown in Fig. 1.

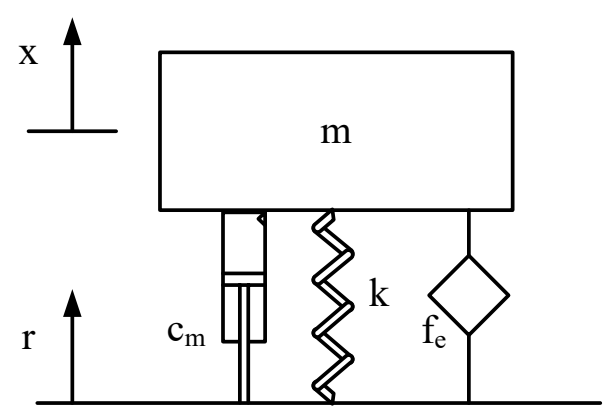

Fig. 1. SDOF base-excited oscillator

The motion equation of the system has form [1]

$$
m \ddot{x}+\left(c_{m}+c_{e}\right)(\dot{x}-\dot{r})+k(x-r)=0,
$$

in which $m, k$ and $c_{m}$ respectively as the mass, the stiffness and the mechanical damping of spring-mass-damper system, $c_{e}$ is the electrical damping provided by the electromotive force $f_{e}$. It is noted that the useful vibration mostly depends on the electrical damping. Denote $r$ and $x$ as the absolute displacements of the foundation and the oscillator's mass, respectively. The non-dimensional form of (1) is

$$
\ddot{x}+2\left(\zeta_{m}+\zeta_{e}\right) \omega_{n}(\dot{x}-\dot{r})+\omega_{n}^{2}(x-r)=0,
$$

where $\omega_{n}=\sqrt{k / m}$ is the natural frequency, $\zeta_{m}=c /\left(2 m \omega_{n}\right)$ and $\zeta_{e}=c_{e} /\left(2 m \omega_{n}\right)$ respectively are the mechanical and electrical damping ratios.

The actual useful vibration is expressed by the average power extracted by the electrical load

$$
P=\frac{1}{T} \int_{0}^{T} f_{e}(\dot{x}-\dot{r}) d t=\frac{2 m \omega_{n}}{T} \int_{0}^{T} \zeta_{e}(\dot{x}-\dot{r})^{2} d t,
$$

where $T$ is a certain simulation time. Under harmonic base excitation with flat acceleration spectrum, consider the harmonic response at steady state, the optimal electrical damping ratio $\zeta_{e}$ has been derived as [1]

$$
\zeta_{e}^{o p t}=\zeta_{m}
$$

The existence of the optimal $\zeta_{e}$ is explained as follows. If $\zeta_{e}$ is too large, the relative velocity is suppressed that reduces the value of (3). Conversely, $\zeta_{e}$ is too small, the vibration can be large but the useful vibration in (3) once again is reduced. 
In this paper, we consider the on-off electrical damping in the form

$$
\begin{aligned}
& \zeta_{e}=\gamma \zeta_{e}^{o p t}, \\
& \gamma=\left[\begin{array}{ll}
\gamma_{h} & \text { certain condition } \\
\gamma_{l} & \text { otherwise }
\end{array}\right.
\end{aligned}
$$

in which $\gamma_{h}$ and $\gamma_{l}$, respectively, are the on-value and off-value of gain of on-off electrical damping. If $\gamma_{h}=\gamma_{l}=1$, we return to the constant passive damping.

\section{MAXIMIZATION OF USEFUL VIBRATION}

Let us consider a class of on-off damping controller, which switches the damping level from high to low and back at fixed times every half period of each given frequency [9]. This controller is quite general that can be use not only to suppress useless vibration but also to amplify useful vibration. Fig. 2 illustrates the switching law of mentioned controller, where $t_{1}$ is the switching time from the high to low, $t_{2}$ is the back switching time and $\Omega$ is the excitation frequency. For all permissible switching times, the controller can be optimized to maximize the useful vibration.

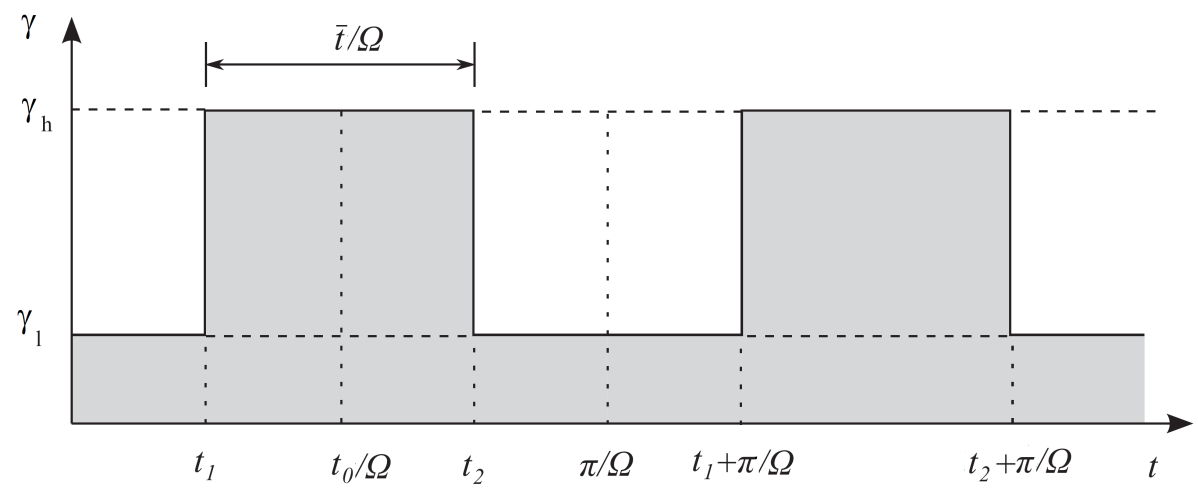

Fig. 2. Illustration of switching times over an excitation period

To simplify the solution, the paper [9] introduced the normalized damping width $\bar{t}$ and the normalized centre of damping peak, $t_{0}$. Both are illustrated in Fig. 2 and nondimensional. All permissible switching times can be described by these two parameters. As mentioned in [1], the acceleration spectrum of vibration sources commonly available in daily lives is relatively flat with frequency. Therefore, we consider the foundation displacement in the form

$$
r=\frac{\Delta}{\Omega^{2}} \sin \Omega t
$$

in which $\Delta$ is the acceleration constant amplitude. Let the relative displacement be written in the approximated form

$$
x-r \approx a_{1} \cos \Omega t+b_{1} \sin \Omega t .
$$


The solution in [9] gives

$$
\begin{aligned}
& a_{1}=\frac{-2 \Delta \omega_{n} \Omega\left(\zeta_{t}+\zeta_{s} \cos 2 t_{0}\right)}{4 \omega_{n}^{2} \Omega^{2}\left(\zeta_{t}^{2}-\zeta_{s}^{2}\right)+\left(\omega_{n}^{2}-\Omega^{2}\right)^{2}} \\
& b_{1}=\frac{\Delta\left(\omega_{n}^{2}-\Omega^{2}-2 \omega_{n} \Omega \zeta_{s} \sin 2 t_{0}\right)}{4 \omega_{n}^{2} \Omega^{2}\left(\zeta_{t}^{2}-\zeta_{s}^{2}\right)+\left(\omega_{n}^{2}-\Omega^{2}\right)^{2}}
\end{aligned}
$$

in which, we denote

$$
\zeta_{t}=\left(\frac{\gamma_{h}-\gamma_{l}}{\pi} \bar{t}+\gamma_{l}\right) \zeta_{e}^{o p t}+\zeta_{m}, \quad \zeta_{s}=\frac{\gamma_{h}-\gamma_{l}}{\pi} \zeta_{e}^{o p t} \sin \bar{t}
$$

Over one vibration period, substitute $T=2 \pi / \Omega$ into (3) give the useful vibration in form

$$
P=\frac{m \omega_{n} \Omega}{\pi} \int_{0}^{2 \pi / \Omega} \zeta_{e}(\dot{x}-\dot{r})^{2} d t
$$

From the motion equation (2), we have

$$
-\omega_{n} \zeta_{e}(\dot{x}-\dot{r})=\ddot{x}+2 \zeta_{m} \omega_{n}(\dot{x}-\dot{r})+\omega_{n}^{2}(x-r)
$$

which changes (11) to

$$
P=\frac{-m \Omega}{2 \pi} \int_{0}^{2 \pi / \Omega}\left(\ddot{x}(\dot{x}-\dot{r})+\omega_{n}^{2}(x-r)(\dot{x}-\dot{r})+2 \zeta_{m} \omega_{n}(\dot{x}-\dot{r})^{2}\right) d t
$$

Substituting the harmonic forms (6) and (7) into (13) and simplifying give

$$
P=\frac{-m \Omega}{2}\left(\Delta a_{1}+2 \Omega \zeta_{m} \omega_{n}\left(a_{1}^{2}+b_{1}^{2}\right)\right)
$$

Substituting (8) and (9) into (14), some manipulations yields

$$
\begin{aligned}
& P=\frac{m \Delta^{2} \alpha^{2}}{\omega_{n}\left(4 \alpha^{2}\left(\zeta_{t}^{2}-\zeta_{s}^{2}\right)+\left(1-\alpha^{2}\right)^{2}\right)} \times \\
& \left(\zeta_{t}-\zeta_{m}+\zeta_{s} \frac{\cos 2 t_{0}\left(4 \alpha^{2}\left(\zeta_{t}^{2}-\zeta_{s}^{2}\right)+\left(1-\alpha^{2}\right)^{2}-8 \alpha^{2} \zeta_{t} \zeta_{m}\right)+2 \zeta_{m} \alpha\left(1-\alpha^{2}\right) \sin 2 t_{0}-8 \zeta_{m} \zeta_{s} \alpha^{2}}{4 \alpha^{2}\left(\zeta_{t}^{2}-\zeta_{s}^{2}\right)+\left(1-\alpha^{2}\right)^{2}}\right),
\end{aligned}
$$

in which, the normalized frequency is introduced

$$
\alpha=\frac{\Omega}{\omega_{n}} .
$$

It is important to noted that, in the passive case, we have

$$
\gamma_{h}=\gamma_{l}=1, \quad \zeta_{s}=0,
$$


which turns the solution (15) to the one presented in [1]. Otherwise, in the on-off electrical damping case, the problem now is to maximize $P$, which is a function of two variables $\bar{t}$ and $t_{0}$. By using the Cauchy-Schwarz inequality, it is not difficult to check that

$$
\begin{aligned}
& P\left(\bar{t}, t_{0}\right) \leq P_{0}(\bar{t})=\frac{m \Delta^{2} \alpha^{2}}{\omega_{n}\left(4 \alpha^{2}\left(\zeta_{t}^{2}-\zeta_{s}^{2}\right)+\left(1-\alpha^{2}\right)^{2}\right)} \times \\
& \left(\zeta_{t}-\zeta_{m}-\zeta_{s} \frac{8 \zeta_{m} \zeta_{s} \alpha^{2}-\sqrt{\left(4 \alpha^{2}\left(\zeta_{t}^{2}-\zeta_{s}^{2}\right)+\left(1-\alpha^{2}\right)^{2}-8 \alpha^{2} \zeta_{t} \zeta_{m}\right)^{2}+4 \zeta_{m}^{2} \alpha^{2}\left(1-\alpha^{2}\right)^{2}}}{4 \alpha^{2}\left(\zeta_{t}^{2}-\zeta_{s}^{2}\right)+\left(1-\alpha^{2}\right)^{2}}\right) .
\end{aligned}
$$

The equality holds when

$$
\begin{aligned}
\cos 2 t_{0 \_o p t} & =\frac{4 \alpha^{2}\left(\zeta_{t}^{2}-\zeta_{s}^{2}\right)+\left(1-\alpha^{2}\right)^{2}-8 \alpha^{2} \zeta_{t} \zeta_{m}}{\sqrt{\left(4 \alpha^{2}\left(\zeta_{t}^{2}-\zeta_{s}^{2}\right)+\left(1-\alpha^{2}\right)^{2}-8 \alpha^{2} \zeta_{t} \zeta_{m}\right)^{2}+4 \zeta_{m}^{2} \alpha^{2}\left(1-\alpha^{2}\right)^{2}}}, \\
\sin 2 t_{0 \_o p t} & =\frac{2 \zeta_{m} \alpha\left(1-\alpha^{2}\right)}{\sqrt{\left(4 \alpha^{2}\left(\zeta_{t}^{2}-\zeta_{s}^{2}\right)+\left(1-\alpha^{2}\right)^{2}-8 \alpha^{2} \zeta_{t} \zeta_{m}\right)^{2}+4 \zeta_{m}^{2} \alpha^{2}\left(1-\alpha^{2}\right)^{2}}},
\end{aligned}
$$

where $t_{0 \_ \text {opt }}$ is the optimal value of $t_{0}$. The problem now is to find the maximum of a single-variable function $P_{0}$ in a fixed interval $(0 \leq \bar{t} \leq \pi)$

$$
P_{M}=\max _{0 \leq \bar{t} \leq \pi} P_{0}(\bar{t})
$$

where $P_{M}$ is the maximum useful vibration can be extracted by on-off electrical damping. It is noted that maximizing a single-variable function in a bound interval can be done by many efficient algorithms.

\section{COMPARISONS BETWEEN ON-OFF DAMPING AND PASSIVE DAMPING}

Fig. 3 shows some plots of the normalized useful vibration $P \omega_{n} / m / \Delta^{2}$ in the frequency domain for varying values of higher gain $\gamma_{h}$ and lower gain $\gamma_{l}$. It is seen that the useful vibration can be amplified remarkable by the on-off electrical damping in comparison with the optimal passive electrical damping. The peak of useful vibration can not be heightened. This conclusion can be drawn from (18) as follows. The peak useful vibration of the passive case is obtained when $\alpha=1$ [1]. Substitute $\alpha=1$ into (18) we have

$$
\left.P_{0}(\bar{t})\right|_{\alpha=1}=\frac{m \Delta^{2}}{4 \omega_{n}\left(\zeta_{t}^{2}-\zeta_{s}^{2}\right)}\left(\zeta_{t}-\zeta_{m}-\zeta_{s} \frac{2 \zeta_{m} \zeta_{s}-\left|\zeta_{t}^{2}-\zeta_{s}^{2}-2 \zeta_{t} \zeta_{m}\right|}{\zeta_{t}^{2}-\zeta_{s}^{2}}\right) .
$$




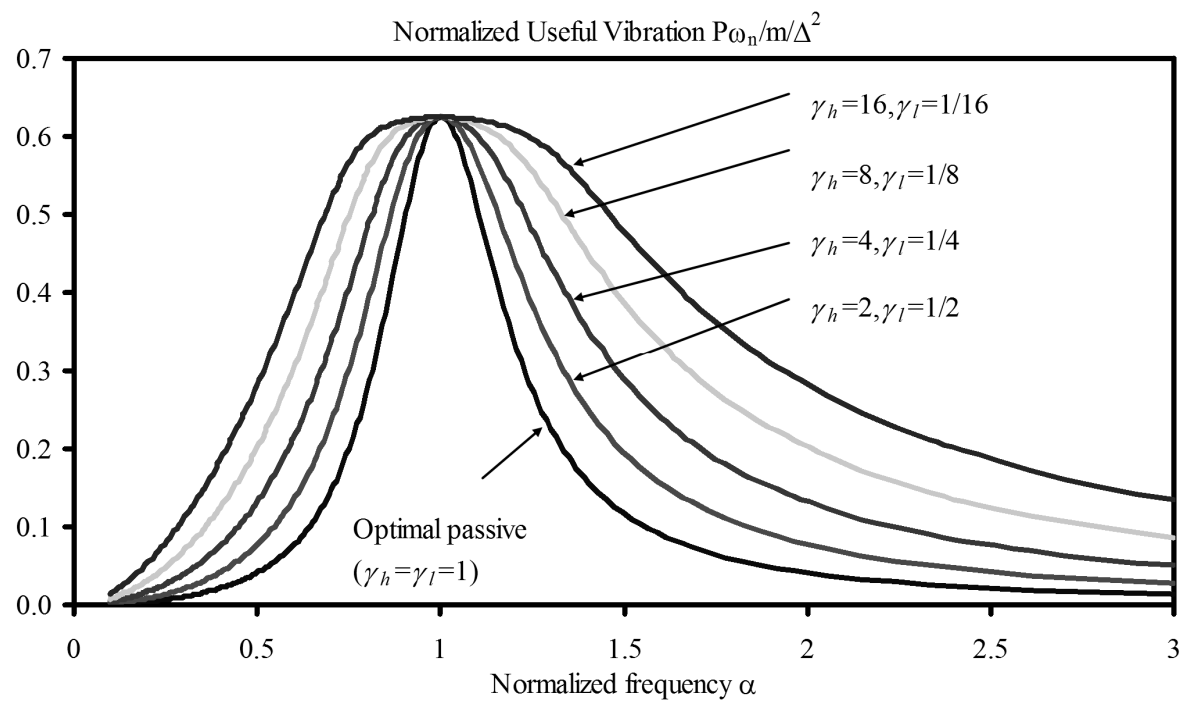

Fig. 3. Normalized useful vibration versus normalized frequency for varying gains and for $\zeta_{m}=0.1$

After some manipulations, we simplify (21) as

$$
\left.\frac{\omega_{n} P_{0}(\bar{t})}{m \Delta^{2}}\right|_{\alpha=1}= \begin{cases}\frac{\left(\zeta_{t}-\zeta_{s}\right)-\zeta_{m}}{4\left(\zeta_{t}-\zeta_{s}\right)^{2}} & \zeta_{t}^{2}-\zeta_{s}^{2}-2 \zeta_{t} \zeta_{m} \geq 0 \\ \frac{\left(\zeta_{t}+\zeta_{s}\right)-\zeta_{m}}{4\left(\zeta_{t}+\zeta_{s}\right)^{2}} & \zeta_{t}^{2}-\zeta_{s}^{2}-2 \zeta_{t} \zeta_{m}<0\end{cases}
$$

It is noted that for any $u$ we have

$$
\frac{u-\zeta_{m}}{u^{2}} \leq \frac{1}{4 \zeta_{m}}
$$

The equality holds when

$$
u=2 \zeta_{m}
$$

In (22), if we substitute $u$ by $\zeta_{t}-\zeta_{s}$ or $\zeta_{t}+\zeta_{s}$, we have

$$
\left.\frac{\omega_{n} P_{0}(\bar{t})}{m \Delta^{2}}\right|_{\alpha=1} \leq \frac{1}{16 \zeta_{m}}
$$

Because the value $1 / 16 / \zeta_{m}$ is the peak of normalized useful vibration in the passive case, the formula (24) implies that the on-off damping can not heighten the peak of useful vibration as shown in Fig. 3.

However, it is also seen in Fig. 3, the width of the curve can be increased, which implies the useful vibration can be amplified in a wider frequency range. The effects of gains are simple: the on-value should be as large as possible while the off-value should be as small as possible.

To see the effect of on-off damping in the limit case, let us consider the zero value of off-gain $\left(\gamma_{l}=0\right)$ and the very large value of on-gain, i.e $\gamma_{h}$ increases to a very large value. 
The normalized useful vibration $P \omega_{n} / m / \Delta^{2}$ is plotted versus the normalized frequency $\alpha$ in Fig. 4.

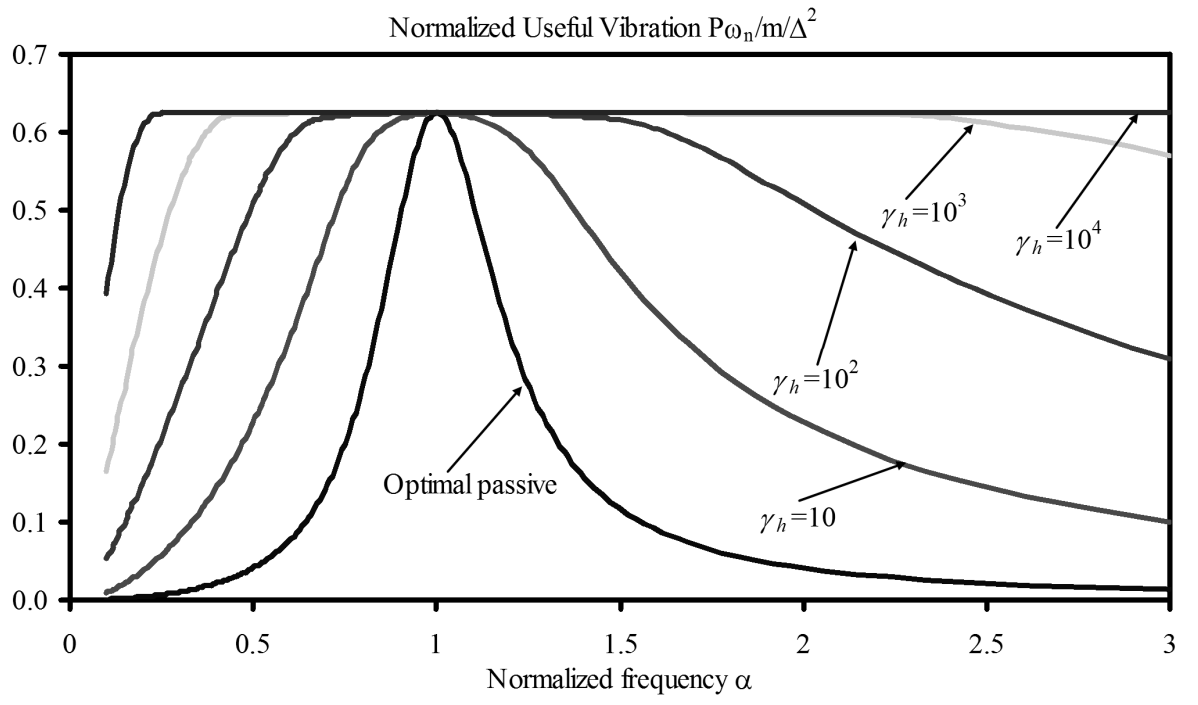

Fig. 4. Normalized useful vibration versus normalized frequency for $\zeta_{m}=0.1, \gamma_{l}=0$ and $\gamma_{h}$ tends to infinity

It is seen that the useful vibration tends to the maximum one of the passive case in a wider range of frequency. The same proof can be done as above when we observe (10) and (18). From (10), when $\zeta_{l}=0$ and $\zeta_{h}$ tends to infinity, two terms $\zeta_{t}$ and $\zeta_{s}$ also tend to infinity. The expression (18) reduces to

$$
\lim _{\substack{\zeta_{h} \rightarrow \infty \\ \zeta_{l}=0}} P_{0}(\bar{t})=\frac{m \Delta^{2}}{4 \omega_{n}\left(\zeta_{t}^{2}-\zeta_{s}^{2}\right)}\left(\zeta_{t}-\zeta_{m}-\zeta_{s} \frac{2 \zeta_{m} \zeta_{s}-\left|\zeta_{t}^{2}-\zeta_{s}^{2}-2 \zeta_{t} \zeta_{m}\right|}{\zeta_{t}^{2}-\zeta_{s}^{2}}\right) .
$$

The expression (26) completely coincides with (21). From (24) we also have

$$
\lim _{\substack{\zeta_{h} \rightarrow \infty \\ \zeta_{l}=0}} \frac{\omega_{n} P_{0}(\bar{t})}{m \Delta^{2}} \leq \frac{1}{16 \zeta_{m}} .
$$

The value $1 / 16 / \zeta_{m}$ is indeed the peak of useful vibration in the passive case in seen in Figs. 3 and 4. The difference between (25) and (27) is that (27) holds for all frequencies.

\section{CONCLUSIONS}

This paper considers the problem of amplifying useful vibration from a base-excited oscillator with on-off electrical damping. We derive the theoretical solution of the maximum available useful vibration can be extracted. The on-off damping can not heighten the peak of useful vibration in the frequency domain in comparison with optimal passive damping. However the larger on-damping and smaller off-damping can widen the useful vibration curve in the frequency domain. Moreover, in the theoretical limit case, 
if the on-damping tends to infinity and off-damping is zero, the simple solution shows that, the peak of useful vibration in the passive case can be extracted in all frequency by the on-off damping. This conclusion opens the opportunities to find the future practical on-off damping controller to extract remarkable useful vibration.

\section{ACKNOWLEDGMENT}

This paper is funded by Vietnam National Foundation for Science and Technology Development (NAFOSTED) under grant number "08/2018/TN".

\section{REFERENCES}

[1] W.-C. Tai and L. Zuo. On optimization of energy harvesting from base-excited vibration. Journal of Sound and Vibration, 411, (2017), pp. 47-59. https://doi.org/10.1016/j.jsv.2017.08.032.

[2] F. Casciati, G. Magonette, and F. Marazzi. Technology of semiactive devices and applications in vibration mitigation. John Wiley \& Sons, (2006).

[3] S. M. Savaresi, C. Poussot-Vassal, C. Spelta, O. Sename, and L. Dugard. Semi-active suspension control design for vehicles. Butterworth-Heinemann (UK), (2010).

[4] E. Lefeuvre, A. Badel, C. Richard, and D. Guyomar. Piezoelectric energy harvesting device optimization by synchronous electric charge extraction. Journal of Intelligent Material Systems and Structures, 16, (10), (2005), pp. 865-876. https://doi.org/10.1177/1045389X05056859.

[5] D. Guyomar, A. Badel, E. Lefeuvre, and C. Richard. Toward energy harvesting using active materials and conversion improvement by nonlinear processing. IEEE Transactions on Ultrasonics, Ferroelectrics, and Frequency Control, 52, (4), (2005), pp. 584-595. https://doi.org/10.1109/tuffc.2005.1428041.

[6] H. Ji, J. Qiu, P. Xia, and D. Inman. Analysis of energy conversion in switched-voltage control with arbitrary switching frequency. Sensors and Actuators A: Physical, 174, (2012), pp. 162-172. https://doi.org/10.1016/j.sna.2011.11.004.

[7] F. Di Monaco, M. G. Tehrani, S. J. Elliott, E. Bonisoli, and S. Tornincasa. Energy harvesting using semi-active control. Journal of Sound and Vibration, 332, (23), (2013), pp. 6033-6043. https://doi.org/10.1016/j.jsv.2013.06.005.

[8] G. Caruso, S. Galeani, and L. Menini. Semi-active damping and energy harvesting using an electromagnetic transducer. Journal of Vibration and Control, 24, (12), (2018), pp. 2542-2561. https://doi.org/10.1177/1077546316688993.

[9] J. N. Potter, S. A. Neild, and D. J. Wagg. Generalisation and optimisation of semi-active, onoff switching controllers for single degree-of-freedom systems. Journal of Sound and Vibration, 329, (13), (2010), pp. 2450-2462. https://doi.org/10.1016/j.jsv.2009.12.011.

[10] L. D. Viet and P. T. T. My. Theoretical upper and lower bounds of the performance of an on-off damping dynamic vibration absorber attached to a multi-degree-of-freedom system. Journal of Vibration and Acoustics, 141, (3), (2019). https://doi.org/10.1115/1.4042278.

[11] L. D. Viet and P. T. T. My. Lower bound of performance index of an on-off damper in a state-space system. Proceedings of the Institution of Mechanical Engineers, Part C: Journal of Mechanical Engineering Science, 233, (12), (2019), pp. 4288-4298. https://doi.org/10.1177/0954406218819028.

[12] L. D. Viet and N. T. Kien. Lower bound of performance index of anti-sway control of a pendulum using on-off damping radial spring-damper. Vietnam Journal of Mechanics, 41, (2), (2019), pp. 193-201. https://doi.org/10.15625/0866-7136/13625. 